8. Manji RA, Grocott HP, Leake J, et al. Seizures following cardiac surgery: the impact of tranexamic acid and other risk factors. Can J Anaesth. 2012; 59(1):6-13.

9. Murkin JM, Falter F, Granton J, Young B, Burt C, Chu M. High-dose tranexamic acid is associated with nonischemic clinical seizures in cardiac surgical patients. Anesth Analg. 2010;110(2):350-353.

10. Kalavrouziotis D, Voisine P, Mohammadi S, Dionne $\mathrm{S}$, Dagenais F. High-dose tranexamic acid is an independent predictor of early seizure after cardiopulmonary bypass. Ann Thorac Surg. 2012;93(1):148-154.

11. Montes FR, Pardo DF, Carreno M, Arciniegas C, Dennis RJ, Umana JP. Risk factors associated with postoperative seizures in patients undergoing cardiac surgery who received tranexamic acid: a casecontrol study. Ann Card Anaesth. 2012;15(1):6-12.

12. Brown EN, Lydic R, Schiff ND. General anesthesia, sleep, and coma. N Engl J Med. 2010;
363(27):2638-2650.

13. Hemmings HC Jr, Akabas MH, Goldstein PA, Trudell JR, Orser BA, Harrison NL. Emerging molecular mechanisms of general anesthetic action. Trends Pharmacol Sci. 2005;26(10):503-510.

14. Antognini JF, Schwartz K. Exaggerated anesthetic requirements in the preferentially anesthetized brain. Anesthesiology. 1993;79(6):1244-1249.

15. Brown EN, Purdon PL, Van Dort CJ. General anesthesia and altered states of arousal: a systems neuroscience analysis. Annu Rev Neurosci. 2011;34:601-628.

16. Schwartzkroin PA. Basic mechanisms of epileptogenesis. In: Wyllie E, ed. The Treatment of Epilepsy. Philadelphia, Pennsylvania, USA: Lea and Febiger; 1993:83-98.

17. Mirsattari SM, Sharpe MD, Young GB. Treatment of refractory status epilepticus with inhalational anesthetic agents isoflurane and desflurane. Arch Neurol. 2004;61(8):1254-1259.
18. Bai D, Pennefather PS, MacDonald JF, Orser BA. The general anesthetic propofol slows deactivation and desensitization of GABA(A) receptors. J Neurosci. 1999;19(24):10635-10646.

19. Ching S, Purdon PL, Vijayan S, Kopell NJ, Brown EN. A neurophysiological-metabolic model for burst suppression. Proc Natl Acad Sci U S A. 2012; 109(8):3095-3100.

20. Furtmüller R, et al. Tranexamic acid, a widely used antifibrinolytic agent, causes convulsions by a gamma-aminobutyric acid(A) receptor antagonistic effect. J Pharmacol Exp Ther. 2002;301(1):168-173.

21. Neville MJ, Butterworth J, James RL, Hammon JW, Stump DA. Similar neurobehavioral outcome after valve or coronary artery operations despite differing carotid emoblic counts. J Thorac Cardiovasc Surg. 2001;121(1):125-136.

22. Makarovsky I, et al. Strychnine-a killer from the past. Isr Med Assoc J. 2008;10(2):142-145.

\title{
Are epsins a therapeutic target for tumor angiogenesis? \\ Nancy Klauber-DeMore
}

Department of Surgery, University of North Carolina at Chapel Hill, Chapel Hill, North Carolina, USA.

\begin{abstract}
Solid tumor growth requires the formation of new blood vessels to supply nutrients and oxygen to the malignant cells; one approach to cancer therapy is to block this process by inhibiting VEGF signaling. In this issue of the JCI, Pasula et al. demonstrate a surprising role of epsins - proteins involved in endocytosis - in tumor angiogenesis via their modulation of VEGF signaling. Their findings suggest that these proteins might represent a new target for the development of cancer therapeutics.
\end{abstract}

Angiogenesis is the formation of new capillary blood vessels and is a critical component of solid tumor growth (1). Once a new tumor reaches just a few cubic millimeters in size, further growth must be preceded by angiogenesis. Tumor cells secrete soluble factors that stimulate vessel growth and/or suppress factors that prevent angiogenesis. These factors act upon endothelial cells to promote their proliferation and migration, resulting in sprouting and tube formation; those tubes then develop into vessels.

Although tumor angiogenesis can be understood as a process required to sustain a cancer's blood supply, the vascular network induced as a result of tumor angiogenesis is highly aberrant, altering the tumor microenvironment and profoundly influencing the manner in which cancers grow, escape the host's immune system,

Conflict of interest: The author has declared that no conflict of interest exists.

Citation for this article: J Clin Invest. 2012; 122(12):4341-4343. doi:10.1172/JCI66171. and metastasize (2). Unlike the organized microvasculature of normal tissue, tumor microvessels are dilated and tortuous, with disorganized patterns of interconnection and branching (3). The erratic tumor vasculature and the resultant hypoxia have additional consequences for tumors: cancer cells undergo epigenetic changes in hypoxic conditions that accelerate their malignant phenotype and the epithelial-to-mesenchymal transition, producing a greater metastatic potential (2). In addition, the cytotoxic functions of immune cells that infiltrate a tumor are compromised in hypoxic and low $\mathrm{pH}$ conditions, further contributing to the malignant phenotype (4).

\section{VEGF family}

An essential mediator of angiogenesis is the VEGF family, which consists of five family members of secreted proteins (VEGFA, VEGFB, VEGFC, VEGFD, VEGFE, and PDGF) (5), that bind and activate three receptor tyrosine kinases (VEGFR1, -2, and -3) (6) which in turn mediate cell sig- naling. VEGFR2 is the key mediator of VEGF-stimulated tumor angiogenesis. When VEGF ligands VEGFR2, the receptor is phosphorylated and activates downstream signaling molecules, resulting in endothelial cell proliferation, migration, tube formation, and the induction of antiapoptotic gene expression (7). VEGF signaling also causes tortuous vasculature formation and vascular leakage in tumors.

\section{Inhibiting VEGF is a therapeutic strategy to inhibit tumor growth}

With the discovery of VEGF as a major driver of tumor angiogenesis, efforts have focused on the development of therapeutics to inhibit VEGF activity, with the goal of inducing tumor regression by starvation. In 2004, a humanized monoclonal antibody to VEGFA, bevacizumab (Avastin; Genentech), became the first FDAapproved antiangiogenic drug in the United States (8). It was approved as a first-line treatment agent for metastatic colorectal cancer, in combination with 5-fluorouracil (9), and was subsequently approved for treatment of metastatic non-squamouscell lung cancer, breast cancer, and glioblastoma multiforme (10). FDA approval was withdrawn for metastatic breast cancer because follow-up studies failed to show an improvement in overall survival. Additional FDA-approved drugs that block VEGF 
A
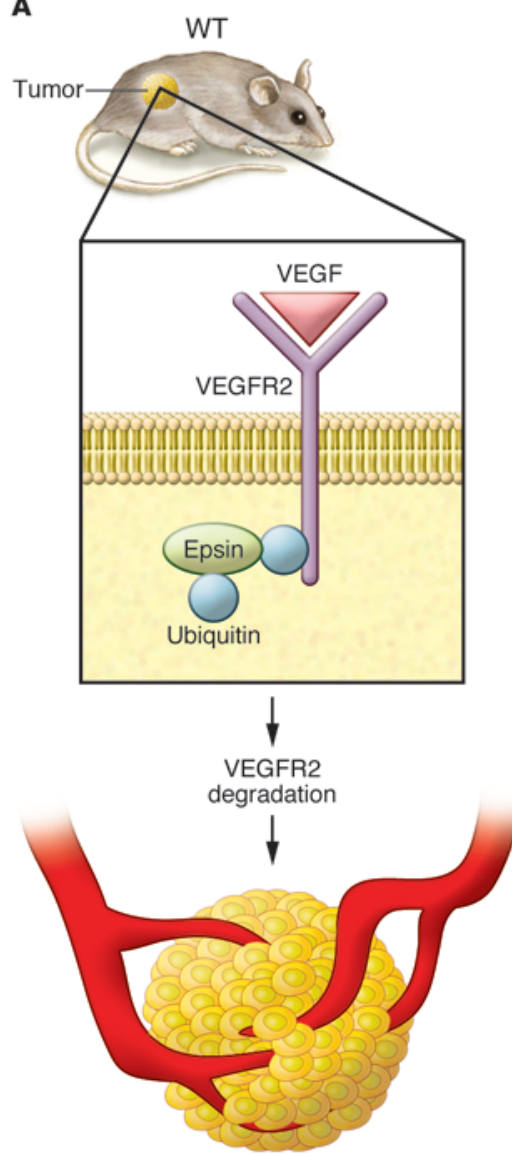

Steady state VEGF/VEGFR2 signaling Functional tumor vasculature Normal tumor growth
B
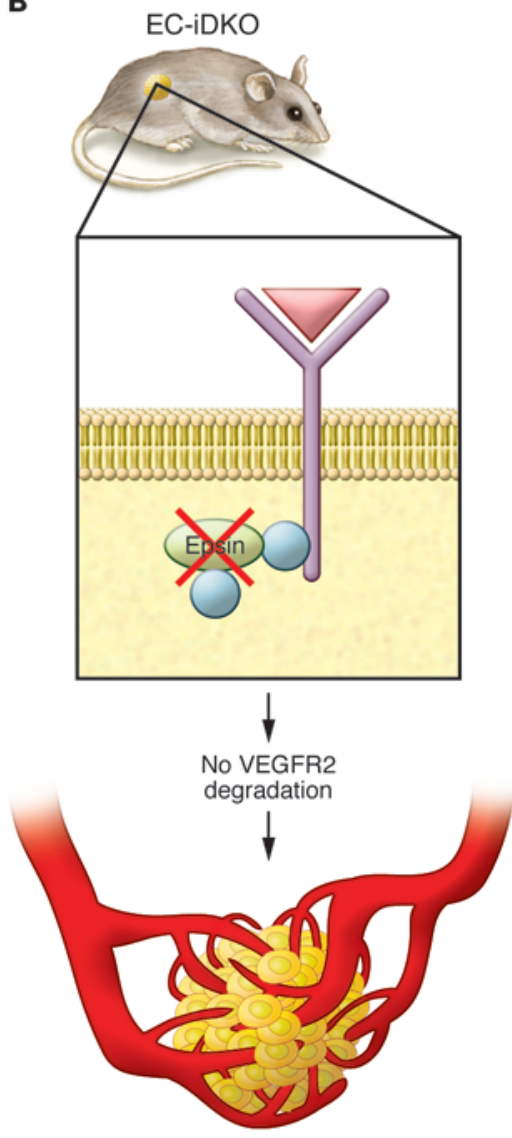

Excessive VEGF/VEGFR2 signaling Nonfunctional tumor vasculature Decreased tumor growth

\section{Figure 1}

Epsins mediate VEGFR2 ubiquitination and endocytosis. (A) VEGFR2 receptors on the plasma membrane undergo ubiquitination as a result of VEGF binding. Ubiquitinated receptors bind to proteins called epsins through a ubiquitin-interacting motif. (B) In EC-iDKO mice, the epsins are inactivated, blocking VEGFR2 receptor degradation. As a result, there is increased VEGF/ VEGFR2 signaling, resulting in aberrant nonfunctional vessels that do not support tumor growth.

signaling are sorafenib and sunitinib, both receptor tyrosine kinase inhibitors, which are administered orally. Sorafenib has been approved for unresectable hepatocellular carcinoma and advanced renal cell carcinoma, whereas sunitinib has been approved for gastrointestinal stromal tumors and metastatic renal cell carcinoma (8) and neuroendocrine tumors (11).

Although VEGF blockade is an attractive strategy to inhibit angiogenesis, an important problem in the field of angiogenesis is that not all patients' tumors respond to anti-VEGF therapy, and of those that respond, most eventually progress (12). Therefore, there is a critical need for better understanding of the molecular regulation of angiogenesis to uncover new therapeutic targets to treat patients with tumors who do not respond to anti-VEGF therapy. The study in this issue by Pasula et al. (13) sheds new light on our understanding of VEGF regulation of angiogenesis via epsin 1 and epsin 2, and uncovers potential new therapeutic targets for inhibiting angiogenesis and tumor growth.

\section{Role of epsins in angiogenesis}

Endocytosis is a complex process in which a portion of the plasma membrane buds inwards toward the cytoplasm to form a small vesicle that contains both cellular membrane and proteins as well as extracellular fluid (14). Several years ago, the epsin family of evolutionarily conserved proteins was discovered on the basis of their ability to bind and link various cell-surface receptors with the endocytic machinery.
Like their binding partners, the epsins were found to be necessary for endocytosis (15, 16). Epsins 1 and 2 are expressed in all tissues (15). Mice lacking either epsins 1 or 2 exhibit no abnormal phenotype, indicating that the proteins have redundant functions. However, epsin 1 and 2 double-KO mice (DKO) die at embryonic day 10 and display profound defects in embryonic vascular development (9). Although an abnormal vascular phenotype is prominent in DKO embryos, it is unclear by which mechanism and in which cell type epsins 1 and 2 are required for angiogenesis. In addition, the role of epsin-regulated angiogenesis in adult animals remained to be addressed.

\section{New experimental findings}

Pasula et al. (13) investigated the role of endothelial epsins 1 and 2 in the adult vascular system by generating a mouse model in which epsins 1 and 2 may be inducibly deleted in endothelial cells (EC-iDKO) under control of tamoxifen and tested their role in tumor growth in both subcutaneously implanted tumors and spontaneous tumor models. Tumors implanted into WT mice grew faster than those in ECiDKO mice, and there was decreased tumor incidence in EC-iDKO in the spontaneous models, demonstrating a role of epsins 1 and 2 in tumor growth. Additionally, the tumor vasculature in EC-iDKO tumors was larger than in WT tumors and was highly disorganized and tortuous with negligible mural cell coverage, suggesting that these tumor vessels were more fragile and immature. The enlarged EC-iDKO tumor vessels were poorly perfused and exhibited extensive extravascular leakage.

Pasula et al. postulate that this nonproductive leaky angiogenesis may lead to impaired oxygen and nutrient supply and thus decreased tumor growth in EC-iDKO mice. They investigated how VEGF signaling was altered and found no difference in VEGF levels between EC-iDKO tumors and WT; however, the level of total and phosphorylated VEGFR2 was strikingly increased. No change was detected for other receptors implicated in angiogenic pathways, including PDGFR- $\beta$, TGF- $\beta$ R 1 , and EGFR in EC-iDKO tumors compared with WT tumors, suggesting that loss of endothelial epsins 1 and 2 selectively affects VEGFR2 signaling. The researchers found that VEGF promotes binding of epsin to VEGFR2 via epsin's ubiquitin interacting motifs. Loss of epsins 1 and 2 specifically impaired endocytosis and 
degradation of VEGFR2 and subsequently resulted in excessive VEGF signaling that compromised tumor vascular function by exacerbating nonproductive leaky angiogenesis (Figure 1).

\section{Conclusions and future directions}

Our understanding of VEGF signaling to date suggests that overexpression of VEGF induces angiogenesis which increases tumor growth. It has been well demonstrated that tumor microvessels are phenotypically abnormal, with increased tortuosity, yet the net result is the promotion of tumor growth and metastasis (2). Pasula et al. (13) have shed new light on the intricacies involved in the regulation of VEGF signaling in tumors, showing that overstimulation of VEGF/VEGFR2 signaling results in dysfunctional leaky vessels that do not sustain tumor growth. Normally, this is prevented by a feedback mechanism whereby VEGF activation of VEGFR2 induces VEGRR2 ubiquitination (mediated by epsins 1 and 2) controlling the extent of VEGF signaling, so that functional vessels are formed. Inhibiting epsins could lead to overstimulation of VEGFR2, with resultant formation of nonfunctional vessels. Thus, the regulation of VEGF ubiquitination of VEGR2 by epsin 1 and epsin 2 suggests a potentially new therapeutic strategy to inhibit tumor growth.

This intriguing finding raises additional questions. First, would blocking epsins be beneficial for patients with tumors that are resistant to anti-VEGF therapies? Resistance to anti-VEGF therapies is associated with increased signaling through parallel pathways (e.g., PDGF) (12). Whether tumors that have become resistant to anti-VEGF therapy have altered the VEGFmediated VEGFR2 ubiquitination pathway is unknown, and further studies of tumors resistant to anti-VEGF therapy are needed to determine whether blocking epsins 1 and 2 would be similarly effective in antiVEGF resistant tumors. Second, what is the effect of the aberrant, leaky tumor vessels on intravasation of tumor cells into the circulation? Because of their leakiness, tumor vessels are a route of tumor dissemination. Are tumor vessels in EC-iDKO mice associated with an increased or decreased rate of tumor metastases? These questions are important in evaluating the value of epsins as a therapeutic target.

One of the advantages of anti-VEGF therapy is normalization of the tumor vasculature, which allows better delivery of chemotherapy to the tumor (3). Although targeting epsins may be a novel therapeutic strategy for inhibiting tumor angiogenesis, a potential disadvantage of this approach is that it is likely that, because there is decreased tumor perfusion, this therapy would not be additive or synergistic in combination with cytotoxic chemotherapy. One could speculate that the aberrant underperfused tumor vessels induced by blocking epsins 1 and 2 would not allow for efficient delivery of chemotherapy to the tumor. This would suggest that blockade of epsins 1 and 2 might be more appropriate in patients who have failed cytotoxic chemotherapy rather than as a first-line therapy. Additional studies are needed to determine whether epsins are a viable therapeutic target for cancer.

\section{Acknowledgments}

This work was supported by NIH grant 1R01CA142657-01A1.

Address correspondence to: Nancy KlauberDeMore, University of North Carolina at Chapel Hill, 170 Manning Drive, Physician's Office Bldg., CB \#7213, Chapel Hill, North Carolina 27599, USA. Phone: 919.966.8007; Fax: 919.966.8806; E-mail: nancy_demore@med.unc.edu.

1. Folkman J. Tumor angiogenesis: therapeutic implications. NEngl J Med. 1971;285(21):1182-1186.

2. Goel S, et al. Normalization of the vasculature for treatment of cancer and other diseases. Physiol Rev. 2011;91(3):1071-1121.

3. Jain RK. Normalizing tumor vasculature with antiangiogenic therapy: a new paradigm for combination therapy. Nat Med. 2001;7(9):987-989.

4. Ganss R, Arnold B, Hammerling GJ. Minireview: overcoming tumor-intrinsic resistance to immune effector function. Eur J Immunol. 2004; 34(10):2635-2641.

5. Ferrara N. VEGF and the quest for tumour angiogenesis factors. Nat Rev Cancer. 2002;2(10):795-803.

6. Olofsson B, et al. Vascular endothelial growth factor B, a novel growth factor for endothelial cells. Proc Natl Acad Sci U S A. 1996;93(6):2576-2581.

7. Ferrara N. Vascular endothelial growth factor: basic science and clinical progress. Endocr Rev. 2004; 25(4):581-611.

8. Oklu R, Walker TG, Wicky S, Hesketh R. Angiogenesis and current antiangiogenic strategies for the treatment of cancer. J Vasc Interv Radiol. 2010; 21(12):1791-1805.

9. Hurwitz H, et al. Bevacizumab plus irinotecan, fluorouracil, and leucovorin for metastatic colorectal cancer. N Engl J Med. 2004;350(23):2335-2342.

10. Grothey A, Galanis E. Targeting angiogenesis: progress with anti-VEGF treatment with large molecules. Nat Rev Clin Oncol. 2009;6(9):507-518.

11. Raymond E, et al. Sunitinib malate for the treatment of pancreatic neuroendocrine tumors. N Engl JMed. 2011;364(6):501-513.

12. Ellis LM, Hicklin DJ. Pathways mediating resistance to vascular endothelial growth factor-targeted therapy. Clin Cancer Res. 2008;14(20):6371-6375.

13. Pasula $S$, et al. Endothelial epsin deficiency decreases tumor growth by enhancing VEGF signaling. J Clin Invest. 2012;122(12):4424-4438.

14. Wendland B. Epsins: adaptors in endocytosis? Nat Rev Mol Cell Biol. 2002;3(12):971-977.

15. Chen H, et al. Epsin is an EH-domain-binding protein implicated in clathrin-mediated endocytosis. Nature. 1998;394(6695):793-797.

16. Wendland B, Steece KE, Emr SD. Yeast epsins contain an essential N-terminal ENTH domain, bind clathrin and are required for endocytosis. $E M B O J$. 1999;18(16):4383-4393. 